\title{
PACTO CON LA RIMA: UN ACERCAMIENTO A LAS SUBJETIVIDADES POLÍTICAS DE JÓVENES LÍDERES RAPEROS QUE PARTICIPAN EN PROCESOS ORGANIZATIVOS LOCALES VINCULADOS AL HIP-HOP EN BOGOTÁ
}

\section{PACT WITH THE RHYME: AN APPROACH TO THE POLITICAL SUBJECTIVITIES FROM THE YOUNG RAPPERS WHO LEADS THE ORGANIZATIONAL LOCAL PROCESS TO BIND WITH THE HIP-HOP IN BOGOTÁ}

\section{David Briceño Arango*}

\section{RESUMEN}

Este artículo explora los vínculos, conexiones y articulaciones presentes entre cultura y política, a partir de algunos procesos organizativos locales liderados por jóvenes raperos en la ciudad de Bogotá, durante el periodo 2014-2015. El objetivo es reconocer cómo estos líderes articulan sus expresiones artísticas con propuestas colectivas que facilitan el acercamiento y el diálogo permanente con las problemáticas locales. En suma, la tesis que se defiende es que la acción política juvenil se expresa a través de experiencias e identidades individuales y colectivas.

PALABRAS CLAVE: COLOMBIA * JOVEN * ANÁLISIS CUALITATIVO * CULTURA * ARTE * COMUNIDAD

\section{ABSTRACT}

This article explore the connections and interaction present between culture and politic, based on some organizational local process leads by rapper young in Bogotá D.C. capital from Colombia, during the period 2014-2015. The objective is recognize how this leads bring together theirs artistic expressions with collective design for make easier the permanent talk and the approach with the local problematics. In short, the thesis that defends is the young political action is expressed go through of the experiences and individual and collectives identities.

KEYWORDS: COLOMBIA * YOUNG * QUALITATIVE ANALYSIS * CULTURE * ART * COMMUNITY 


\section{INTRODUCCIÓN}

El presente artículo surge como resultado de un proceso de investigación desarrollado en el marco de la línea de estudio "Dinámicas Sociales Contemporáneas"1. El tema que articula las reflexiones propuestas, serán las subjetividades políticas que emergen en los procesos organizativos locales de jóvenes líderes raperos en la ciudad de Bogotá, durante el periodo 2014- 2015.

Estos procesos de subjetivación, serán vistos desde las experiencias y procesos de trabajo comunitario que llevan a cabo estos jóvenes a lo largo de sus trayectos en las organizaciones. De este modo, el propósito central del presente texto será reconocer esas subjetividades que se construyen cuando se participa en una experiencia organizativa; se trata de conocer sus valoraciones acerca del trabajo desarrollado con la gente $y$ de las condiciones que limitan o posibilitan el desarrollo de sus propuestas.

La idea central que se defenderá en el presente artículo, es la siguiente: asumir el liderazgo de un proceso de organización comunitaria con sectores populares, implica para un joven, la articulación de sus expresiones artísticas y la puesta en marcha de estrategias no previstas, que deben articularse a las problemáticas e intereses del contexto en donde estos líderes desarrollan su trabajo. Es decir, un joven que tiene la intención de generar acciones a partir de sus intereses, es interpelado constantemente por el contexto en donde pretende actuar y este escenario, posibilita la constitución de subjetividades políticas.

La perspectiva que permitió una mayor comprensión de estos procesos organizativos fue la cualitativa, de orientación reflexiva. En contravía a la mirada positivista que plantea una escisión entre el objeto y el sujeto heredada de las ciencias naturales, se asume la perspectiva reflexiva en la que los objetos

1 Pertenece al programa de Trabajo Social de la Corporación Universitaria Republicana, Colombia. de investigación son el resultado de un arduo proceso de construcción ${ }^{2}$.

En coherencia con la perspectiva asumida, se considera que es posible aproximarse a las subjetividades a través del lenguaje. Estas aparecen en los relatos de los jóvenes y expresan saberes, prácticas, sentidos, deseos y propuestas. Se asume en este texto, que estas voces son representaciones de la realidad, porque el lenguaje no es un reflejo exacto de lo real. Por el contrario, "si la cultura es el fundamento de los relatos y nos movemos en una realidad interpretada [...] entonces solo es posible comprender los discursos" (Puyana, 2013, p. 114). Estos expresan una realidad vivida $e$ interpretada, pero no son la realidad.

La ruta metodológica construida se estructuró en dos niveles: el primero, orientado a conocer las experiencias particulares de las organizaciones que participaron en este proceso, desde el relato mismo de los jóvenes que han liderado estas iniciativas. El segundo, dirigido a comprender cómo estos actores sociales se relacionan con el espacio público, particularmente desde las acciones que desarrollan en el ámbito local y en su participación en algunos escenarios institucionales. En esta búsqueda, se hicieron ocho entrevistas individuales a jóvenes líderes que dinamizan estos procesos de organización local.

El número de jóvenes escogidos no constituye un porcentaje representativo en términos estadísticos. No obstante, la muestra se construyó con un criterio no probabilístico. Se trata de una muestra estructural (Navarrete, 2000) que se construye con sujetos que ocupan un lugar importante en los procesos de organización local, $y$ en virtud de ese lugar, tienen un discurso representativo porque poseen un conocimiento social relevante para dar cuenta de este fenómeno. Los sujetos que participaron en esta experiencia son: jóvenes líderes de colectivos culturales que viven

$2 \quad$ La teoría de la práctica en cuanto práctica recuerda, contra el materialismo positivista, que los objetos de conocimiento son construidos y no pasivamente registrados $y$, contra el idealismo intelectualista que el principio de dicha construcción es el sistema de las disposiciones estructuradas y estructurantes que se constituyen en la práctica (Bourdieu, 2007, p. 85). 
en barrios populares de distintas localidades de Bogotá, tienen una afinidad con el hip hop (ya sea hacia sus líricas, por el graffiti o el break dance) y la mayoría son estudiantes universitarios.

Los colectivos juveniles que acompañaron este proceso investigativo fueron: Corporación Cultural Urban Art, Duncan, Decibeles Récords, Golpe Natural, Slam Painting, Latin Fury, Universal Rimas Colombia, Fundación chocolate y pan con humildad, Organización Cultura Razón y Realidad. Estas organizaciones se ubican en barrios populares de Bogotá, en las siguientes localidades: Santafé, Rafael Uribe Uribe, Los Mártires, La Candelaria, Kennedy y Chapinero.

Los lugares donde los jóvenes desarrollan su trabajo se caracterizan por una subordinación estructural en términos del acceso a la educación, las posibilidades de un trabajo bien remunerado y la seguridad. En Bogotá, según el informe de calidad de vida (Equipo ¿Bogotá cómo vamos?, 2016) realizado por la Alcaldía Distrital, aumentaron los programas de educación superior (de 326 124, en 2010 a 465 501, en 2015); sin embargo, solo la mitad de quienes terminan el bachillerato acceden inmediatamente después de que se gradúan. Si ponemos en contexto este dato, se hace una imagen de las limitaciones socioeconómicas de las personas que viven en barrios marginales donde tienen lugar las experiencias que señalan estos jóvenes.

La cuarta parte de los jóvenes está al margen de las oportunidades cuyo acceso exige recursos monetarios. Esto les impide conseguir determinadas opciones de vida $y$, en consecuencia, frustraría sus posibilidades de desarrollo personal. Los cálculos más recientes sobre cambios en el ingreso por habitante en Bogotá y niveles de concentración de este ingreso entre los grupos sociales indican una tendencia al agravamiento de esta situación y, por tanto, a una exclusión creciente $^{3}$ (Castaño, 2008).

3 Las cifras que sustentan estos planteamientos se encuentran en el informe elaborado
Por esta razón, estás experiencias colectivas resultan pertinentes, ya que en estos escenarios ocurren luchas por la distribución equitativa de bienes materiales y simbólicos. El presente texto se organiza en tres partes: la primera, ubica el campo problemático en el que se inscribe el presente estudio dentro de las ciencias sociales; la segunda parte, presenta la trayectoria de las organizaciones mencionadas anteriormente $y$ sus procesos de trabajo comunitario, desde el relato de los jóvenes que asumen el liderazgo de estos procesos; $y$ en la tercera parte, se proponen reflexiones frente a lo encontrado en las narrativas.

\section{CAMPO PROBLEMÁTICO}

La presente reflexión tiene el interés de explorar las relaciones, vínculos y articulaciones presentes entre jóvenes, cultura y política. En las ciencias sociales se plantean cada vez más esfuerzos por reconocer cómo la acción política juvenil ya no transcurre exclusivamente en aquellos espacios convencionales donde se hace la política (el Estado, sus instituciones o partidos políticos); por el contrario, hoy lo político adquiere mayor protagonismo en espacios donde se entrecruza lo cultural, lo social e inclusive, lo personal. Por ejemplo, se observa cómo temas que antes eran privados (el género, la división del trabajo en la familia $y$ las condiciones de vida) se han politizado $y$ desplazado a otros escenarios.

¿Por qué se produce este cambio? Al leer sobre la juventud y su relación con la acción colectiva, saltan a la vista referencias que señalan un contexto cada vez más globalizado e interconectado, no solo económica y políticamente, sino también, a través del flujo de símbolos que transforman nuestras visiones e imaginarios del mundo. Según Zarzuri (2005), se vive un momento en el que "los conceptos existentes adquieren nuevas formas; donde la cultura, lo social, lo político por señalar algunas

por Econometría S.A para el Departamento Administrativo de Acción Comunal Distrital (DAAC) "Situación socioeconómica de los jóvenes en Bogotá” Bogota 2003, el cual incluye una línea base para la juventud bogotana. 
dimensiones, mutan, se transforman, se diversifican o minimizan" (p. 4).

Frente a este contexto de cambio, resulta indispensable la reflexión por lo político $y$ de manera especial, por la relación entre los jóvenes y la política ¿Qué es la política en las sociedades contemporáneas? ¿Son los jóvenes actores políticos? Estas preguntas necesitan responderse a la luz de dichos cambios, pues no resulta sensato definir la política hoy sin reconocer las transformaciones reales $y$ conceptuales que ha sufrido esta categoría. Autores como Darío Restrepo (2003) y Norbert Lechner (1996), plantean que hoy la política ya no es la principal instancia de orden y articulación de la vida social.

La política ya no es lo que era antes; se abren sus esferas, cambian sus temas, se multiplican sus actores y se ensanchan sus fronteras. Para algunos situados en el extremo derecho e izquierdo del espectro político y social, la política abandona el Estado y se desplaza hacia el mercado y la sociedad. Así es, aunque todavía el Estado es un referente inevitable de las relaciones sociales (Restrepo, 2003, p. 9).

Que la política se desplace del Estado hacia el mercado y la sociedad, es ya un cambio cualitativo que pone en entredicho el lugar en donde esta ocurre hoy. Este cambio, ha dado lugar a espacios más autónomos, pero al mismo tiempo más individualizados, en los que existe una pugna de diferentes actores sociales por reconstruir sentidos alternativos de lo político, en el marco de unas relaciones asimétricas de poder.

Este es el caso de los colectivos juveniles que aparecen en el presente artículo. Estos emergen como consecuencia de un proceso en el que la política se ha desplazado a otros lugares en donde se lucha de distintas formas, por la distribución de bienes materiales, por interpretaciones del mundo y por la reivindicación de unas identidades. Como se observa, los colectivos de jóvenes raperos y raperas "van configurando una geografía grupal: se trata de lugares dispuestos para el encuentro; toman forma gracias a la acción colectiva, y pueden transformase a través del break, el graffiti o el rap; expresiones que dan sentido al lugar tomado por el hoper's en su interacción cotidiana" (Garcés, 2007, p. 11).

En estos colectivos, se materializa la relación que se ha mencionado entre cultura y política. No solo porque estos jóvenes desarrollen prácticas artísticas, sino principalmente porque a partir de estas, proponen procesos colectivos en donde se producen unos significados que pueden tener incidencia ante problemáticas locales. Arturo Escobar (2001), plantea cómo lo cultural puede ser un vehículo de lo político: "la cultura es política porque los significados son elementos constitutivos de procesos que buscan dar nuevas definiciones del poder social. Cuando los movimientos manifiestan conceptos alternativos, desestabilizando significados culturales dominantes, ponen en marcha una nueva política cultural" (Escobar, Alvarez y Dagnino, 2001, p. 19).

No se plantea de entrada que estos colectivos abanderen una nueva política cultural, pero sí son escenarios en donde se está redefiniendo constantemente lo político. Se considera que no es posible entender estas colectividades al margen de esta relación, porque en estos espacios se construyen modos de ser $y$ hacer, relaciones con otros actores $y$ distintas formas de organizar el poder. Si es cierto que lo político se expresa hoy en otros espacios, se comparte la propuesta de Lechner (2002): "necesitamos una redefinición de la política; no en el sentido de una definición taxonómica, sino de una comprensión más cabal de la(s) «lógica(s)» que condicionan la acción política en nuestras democracias" (p. 6) Esta comprensión, desde el punto de vista propio, solo será posible si se reconocen aquellas prácticas emergentes que desbordan la acción del Estado y que según Zemelman (1989), asumen lo político como una la lucha por dar una dirección a la realidad social en el marco de opciones viables.

Desde esta mirada de lo político, son los sujetos sociales quienes en sus prácticas y proyectos podrían instituir rumbos distintos. Por esto resulta importante retomar la propuesta de Reguillo (2000), quien propone pensar a los jóvenes como agentes con capacidad de 
decisión y acción "como sujetos de discurso, con capacidad para apropiarse (y movilizar) los objetos tanto sociales y simbólicos como materiales, es decir, como agentes sociales" (p. 36).

En este sentido, la relación juventud y política, no es excluyente. Si bien, los jóvenes se deben a unas condiciones objetivas que restringen su capacidad de acción, estos luchan por construir alternativas a esa realidad estructurada. Estos dos modos de existencia de lo social (lo instituido e instituyente) se influencian recíprocamente y configuran el escenario propicio para la construcción de subjetividades políticas.

En razón de lo anterior, la construcción de lo político en los jóvenes se vincula con otros ejes que escapan a las formas tradicionales de configurar o construir lo político. En palabras de Reguillo: "la política en los jóvenes pasa por el deseo, la emotividad, la experiencia de un tiempo circular, el privilegio de los significantes sobre los significados, las prácticas arraigadas en el ámbito local que se alimentan incesantemente de elementos de la cultura globalizada" (2000, p. 43). En este sentido, la categoría que permite acercarse a los cambios que ha sufrido lo político en los espacios de acción colectiva juvenil, es la de subjetividades políticas. Se entiende la subjetividad como un:

Conjunto de instancias y procesos de producción de sentido, a través de los cuáles los individuos y los colectivos sociales construyen y actúan sobre la realidad, a la vez que son constituidos como tales. Involucra un conjunto de normas, creencias, lenguajes $y$ formas de aprehender el mundo, conscientes e inconscientes, cognitivas, emocionales, volitivas y eróticas, desde las cuales los sujetos elaboran su experiencia existencial y sus sentidos (González, Aguilera y Torres, 2014, p. 53).

La noción de subjetividad planteada, se sustenta en una idea de sujeto como proceso inacabado como proyecto. Hacerse sujeto en palabras de Touraine (1993), es expresar la voluntad de un individuo para actuar y ser reconocido como actor. Allí el sujeto lucha por desmarcarse de aquellas normas o regulaciones externas que constriñen su libertad sexual, estética o política $y$ plantea proyectos que le den sentido a su estar en el mundo.

En esta dirección, la subjetividad política es un proceso donde el sujeto genera una reflexión constante de sus condiciones de existencia, que le sirven como marco de referencia para actuar en una realidad estructurada $y$ por estructurar. En este proceso de reflexión-acción, los jóvenes no solo desarrollan su trabajo con la gente, sino que se van constituyendo en actores políticos.

\section{SOBRE LAS ORGANIZACIONES SOCIALES DE HIP-HOP Y SUS EXPERIENCIAS}

Como se mencionó al inicio del texto, es a través del lenguaje que se puede acercar a las subjetividades que construyen los jóvenes en estos colectivos. Allí aparecen unos relatos que expresan unas identidades, valoraciones, conocimientos, creencias y prácticas sobre sus trayectos de vida, que podrían hacer visible la relación entre cultura y política.

Estos relatos que expresan la vida de los jóvenes, pueden comprenderse pero sobre todo sentirse, desde la categoría de estilos de vida propuesta por Giddens (1997), entendida como un "conjunto de prácticas más o menos integradas que un individuo adopta no solo porque satisfacen necesidades utilitarias, sino porque dan forma material a una crónica concreta de la identidad de yo" (p. 106). Es decir, los estilos de vida son prácticas hechas rutinas, que van constituyendo lo que yo soy y que están abiertas al cambio en función de esas identidades.

En esta dirección, se busca hacer visible los estilos de vida de estos jóvenes en dos niveles: el primero, orientado a conocer sus experiencias particulares de organización desde el relato, haciendo énfasis en sus motivaciones, líneas de trabajo y planes a futuro. El segundo, dirigido a comprender cómo estos actores sociales comienzan a hacer presencia en el espacio público, a través de las acciones que desarrollan en el ámbito local: procesos de formación, realización de eventos y gestión de recursos con las instituciones. 
El lector debe valorar estos relatos, en clave de cómo los procesos organizativos vinculados al hip-hop, pueden constituir nuevos actores políticos. Un proceso colectivo que inicialmente fue pensado por un joven para promover el hip-hop, no solo termina involucrando las dinámicas del territorio, sino que se constituye en la posibilidad de formación de unos sujetos que comienzan a entender las implicaciones de actuar colectivamente. La apuesta es por ejercicios investigativos donde las voces hagan visible las luchas que emergen allí. En la siguiente sección, se observará las voces de los jóvenes que dinamizan estas organizaciones.

\section{ESCUELA DE FORMACIÓN GOLPE NATURAL}

Esta organización surge como resultado de mi experiencia con el Hip Hop en el centro de Bogotá; allí tuve amigos raperos con los que he creado redes de trabajo, pues en mi barrio no existían espacios que promovieran esta cultura. Entonces, empecé a ver los parchesitos en el barrio que se la pasaban por ahí y eso me motivo a crear el espacio, a abrir los talleres... lo que resultó bastante bueno, porque muchos muchachos con problemáticas serias han llegado y su cotidianidad se ha ido transformando.

Trabajamos con los jóvenes temas que los están comprometiendo; como el servicio militar obligatorio, el consumo de drogas, la sexualidad responsable, el consumo de marcas y el consumo de sustancias.

La experiencia más bonita de ser líder de esta organización, son los mismos jóvenes que le pagan a uno con esa gratitud y con esa capacidad de hacer las cosas. A veces uno piensa que los muchachos del barrio no son capaces de dar mucho, pero dándose esa confianza y ese conocimiento, los muchachos lo pueden respaldar a uno en donde sea. Esa es la experiencia más bonita, ver que ya hay muchachos que te respaldan y que el día que uno se vaya, va a quedar esa semilla como si uno estuviera ahí (Entrevista a joven líder rapero de la localidad Rafael Uribe Uribe, barrio Bravo Páez, 2014).

\section{CORPORACIÓN CULTURAL URBAN ART}

Me conocen como "Mulato" y soy el director-fundador de la Corporación Cultural Urban Art. La escuela más allá de enseñar sobre el hip hop, quiere mostrar que hay otras posibilidades $y$ borrar la estigmatización de que cuando uno vive en un barrio como Egipto, no hay muchas ofertas.

Entre las cosas que nosotros desarrollamos, tenemos un proceso de formación. Urban Art realiza un festival conocido como urban fest, donde se llevan a cabo tres actividades: el RELAMPA RAP: un campeonato relámpago de microfútbol que busca vincular a la comunidad con nuestro género musical, al igual que amenizar el torneo con muestras de grafiti y break dance. DANZA LA CALLE: un evento en el que bailan los B.boy's más importantes de la escena Distrital en el momento, $y$ por último, UN TOQUE POR NUESTROS DERECHOS: donde se hace una convocatoria abierta a la ciudad y se eligen los mejores 8 artistas.

También participamos en convenios o proyectos con instituciones públicas, donde se han planteado dos iniciativas interesantes a nivel Distrital: la alianza de escuelas de formación de hip hop con idARTEs, que vincula a la universidad pública con las escuelas de formación. A través de esta alianza, se logró el diplomado arte, ciudad y pedagogía y el tercer encuentro de escuelas de formación de hip hop en Bogotá (Entrevista a joven líder rapero de la localidad de La Candelaria, barrio Egipto, 2014). 
Mi nombre artístico es Bboy Lascru y soy uno de los líderes de la Escuela. Nuestros procesos están enfocados a niños de 5 años en adelante. La mayoría de niños son de bajos recursos y realizan sus estudios en colegios públicos de barrios cercanos como el Jorge Soto, El corral y La giralda.

Nuestro objetivo es hacer un trabajo social con los niños de la localidad, formar no solo bailarines sino futuros talleristas. Aparte de que vienen a entrenar, tratamos de formarlos como personas, como amigos, como una familia, creando unas redes internas; si vemos que alguien está mal, tratamos de ayudarlo, no podemos dejar que ellos bajen el rendimiento académico, porque nuestro trabajo no es entorpecer el proceso que ellos llevan, sino fortalecerlo (Entrevista a joven líder rapero de la localidad Santafé, Barrios Jorge Soto, El Corral y La Giralda, 2014).

\section{UNIVERSAL RIMAS COLOMBIA}

Soy canta-autora de rap, me conocen como Kapela y pertenezco a Universal Rimas Colombia, una corporación de hip hop que lleva más de 8 años trabajando en formación y producción de eventos. Realizamos eventos en pro del mejoramiento, visibilización y fortalecimiento de nuestros artistas a nivel distrital; Universal, tiene como evento madre UNIDAS POR EL GOLPE: evento dirigido a mujeres que hacen hip hop en cualquiera de las áreas.

Lo más importante de este proceso, es que uno ve la necesidad de transformación desde los chicos, porque uno también fue alumno y estuvo con todas esas ansias de querer trascender, aprender $y$ avanzar. Entonces nosotros vemos en nuestros niños, niñas, jóvenes y mujeres lo que quieren llegar a ser por medio del arte $y$ decidimos apoyarlos.

Cuando vemos a los chicos que ni siquiera sabían coger un micrófono $y$ empiezan a soñar y creer en ellos mismos, eso no tiene precio. Es decir, tu pones una tarima, un sonido o un espacio y una publicidad para ellos y dices: "a continuación fulana o fulano de tal", cuando los ves que se paran en una tarima y se lo sollan, sudan, lloran y les tiembla las piernas, pero que aun así, lo lograron, eso es tremenda satisfacción. Por ejemplo, el chico que empezó con nosotros hace dos años, hoy ya tiene un demo y se comporta como un artista, eso no tiene palabras (Entrevista a joven líder rapera de la localidad de Rafael Uribe Uribe, 2014).

\section{FUNDACIÓN CHOCOLATE Y PAN CON HUMILDAD}

Soy rapero y me conocen como El Hampa Subterráneo. Dirijo la Fundación Chocolate y Pan con Humildad y llevo 10 años radicado en la ciudad de Bogotá. Mi visión del barrio al que llegue, fue como los niños... la marginalidad, el problema de las drogas, del abandono y pues el problema del hambre. Desde ahí, nació el interés de hacer esta obra social.

En este momento solamente salimos a las calles, repartimos chocolate $y$ pan, $y$ les brindamos a los niños un alimento, pero la idea de nosotros es poder sacar los talleres y realizar la parte de la educación y la formación.

La fundación de nosotros tiene alrededor de 1000 niños, la mayoría de estos niños viven en edificios abandonados tomados a 
la fuerza por personas desplazadas, entonces son niños en condiciones muy duras, muy difíciles y la mayoría de sus madres son cabeza de hogar sin muchas alternativas (Entrevista a joven líder rapero de la localidad de los Mártires, 2014).

\section{ORGANIZACIÓN CULTURA RAZÓN Y REALIDAD}

Soy B-boy Smith, bailó Break Dance hace 13 años y hago parte de la organización Cultura, Razón y Realidad. Fui el fundador de dicha organización y tenemos procesos de formación en hip hop, producción de eventos $y$ diferentes actividades encaminadas a la evolución del hip hop.

Nosotros éramos 4 jóvenes que nos reuníamos en un salón comunal a practicar break dance $y$ pues llegaban algunos de los vecinos, en su mayoría niños, y nos pedían que les enseñáramos a bailar. Inicialmente lo hicimos de una manera muy empírica $y$ nos dimos cuenta que el impacto era muy grande, que había más gente interesada. Pero detrás de este interés, comenzamos a ver que nosotros éramos de un barrio vulnerable y que los que vivíamos aquí, estábamos sujetos al consumo de drogas y expuestos a delinquir para poder sobrevivir. Eso fue lo que nos motivó, no tanto a iniciar el grupo de break dance, ni a ser los talleristas de hip hop, sino a profundizar mucho más para realizar un trabajo social real por la comunidad (Entrevista a joven líder rapero de la localidad de Kennedy, barrio Altamar, 2014).

\section{ALGUNAS REFLEXIONES PARA AMPLIAR LA DISCUSIÓN}

Parece haber un elemento común en los testimonios recién citados: los jóvenes raperos y raperas, en sus discursos y prácticas muestran cómo enfrentan colectivamente la existencia $y$ los desafíos de vivir en contextos que están caracterizados por una desigualdad estructural. Con el otro, se comparten las demandas del barrio: "muchos muchachos con problemáticas serias han llegado", "comenzamos a ver que nosotros éramos de un barrio vulnerable". Este ímpetu de acción colectiva, que aún se expresa en los espacios locales populares como resultado de una pertenencia hacia el territorio y a una cultura juvenil globalizada, se pone hoy a prueba, en un momento donde el sujeto atraviesa un proceso de fragmentación e individualización que pone en entredicho su relación con el orden de lo colectivo.

El desasimiento que experimenta el ciudadano, halla su encuadre en una matriz única de sentido: El mercado autorregulado despedaza la conexión con un orden colectivo objetivo, un orden que arde más allá del fuero interno individual pero al que, no obstante, cada ser humano se remite (Perea, 2008, p. 23).

No obstante, resultaría simplista proponer que todos los sujetos están desarticulados o individualizados y no plantean alternativas para contrarrestar este orden. Como se menciona en uno de los apartados, lo político se ha desplazado hoy a otros espacios como resultado de un contexto en el que la globalización es protagonista. Frente a este panorama, Perea (2008) plantea la siguiente interrogante: ¿habrá alguna oportunidad para un nuevo impulso ético de la comunidad en lo urbano popular?

Si se sitúa esta sugerente interrogante en los procesos de organización comunitaria local que desarrollan los sujetos de esta investigación, surgen los siguientes cuestionamientos: ¿qué de comunitario hay en los procesos que estos jóvenes movilizan?, ¿los jóvenes renuncian a su individualidad al liderar un proceso colectivo?, ¿se apropian de lo público?, ¿cómo se da la tensión entre lo individual y lo colectivo en estos escenarios de socialización? Se mostrarán algunas reflexiones desde lo encontrado en las narrativas, en clave de cómo se van configurando nuevas subjetividades políticas. 
a) Cuando se preguntó a los jóvenes sobre las motivaciones que los impulsaron a liderar un proceso de organización, resaltaron varios aspectos. Por un lado, parece evidente que la acción de estos actores sociales se sustenta en un interés particular. En este caso, la afinidad a una cultura juvenil en la que circulan unos símbolos e ideologías sobre la realidad social.

No se puede llegar a afirmar tajantemente que los posicionamientos $\mathrm{u}$ orientaciones de una determinada cultura juvenil, impulsan a estos actores a asumir una experiencia organizativa, pero no hay que perder de vista que los procesos identitarios que se construyen en la mediación de estos sujetos con bienes culturales como la música - por poner un ejemplo importante - podrían afectar la subjetividad de los jóvenes al encontrar semejanzas entre las temáticas sociales abordadas en las canciones $y$ lo que ellos viven en su cotidianidad.

En ese sentido, los jóvenes raperos manifestaron abiertamente que el interés de liderar una organización, surgió como resultado del vínculo estrecho que tenían con el hip-hop. Frases como: "desde muy niña escucho rap y asisto a eventos" (Entrevista a joven rapera, Universal Rimas Colombia, 2014), "toda mi vida he sido muy apasionado por las artes en general y por la música, más que todo enfocada al hip hop" (Entrevista a joven rapero, Decibeles Records, 2014) y "bailó break dance hace 13 años" (Entrevista a joven rapero, Cultura, Razón y Realidad, 2014), muestran el "pacto", usando la imagen con la que se nombra este texto, que existe entre la afinidad a una cultural juvenil que expresa problemáticas de sectores populares, $y$ lo que estos actores pueden llegar a proponer desde sus intereses y destrezas, para contrarrestar estas dinámicas que están presentes en los lugares donde buscan desarrollar su trabajo.

b) Un aspecto que resulta crucial, es la trayectoria de estos jóvenes con el hip-hop. En sus historias particulares, dentro de esta cultura juvenil, construyen un saber valioso que sirve de soporte para liderar un proceso de organización. No es lo mismo iniciar un proceso que vincula a otros contando con unos saberes y destrezas, a desarrollar una iniciativa que busca movilizar personas, sin tener experiencia. Este trayecto es reconocido por los otros y facilita el trabajo con los otros.

Sin embargo, ser portador de un saber o de una destreza artística o cultural, no es garantía suficiente para desarrollar un trabajo comunitario en un contexto particular determinado. Los jóvenes allí, se enfrentan a una disyuntiva entre su interés particular y el interés colectivo; entre sus propios límites, los límites del contexto $y$ las posibilidades que encuentran en este. Los sujetos de este estudio comentan lo siguiente: "empecé a ver los parchesitos en el barrio que se la pasaban por ahí y eso me motivo a crear el espacio" (Entrevista a joven rapero, Golpe Natural, 2014), "eso fue lo que nos motivó, no tanto a iniciar el grupo de break dance, ni a ser los talleristas de hip hop, sino a profundizar mucho más para realizar un trabajo social real por la comunidad" (Entrevista a joven rapero, Cultura, Razón y Realidad, 2014).

El sujeto aquí, desplaza la individualidad en su discurso, por un otro con el que también se siente identificado. Ese otro probablemente comparte, no solo la afinidad hacia el género musical, sino en problemáticas comunes "formamos jóvenes que están involucrados en el consumo de drogas, pues yo también pase por esa experiencia" (Entrevista a joven rapero, Urban Art, 2014).

En relación a los objetivos que orientan el trabajo que desarrollan las organizaciones, resulta interesante que aunque sea lo artístico el motor de estas, ello se cruce con lo social y lo político; allí las fronteras son cada vez más difusas. Objetivos como: "borrar la estigmatización del barrio" (Entrevista a joven rapero, Urban Art, 2014), "formar no solo bailarines sino futuros talleristas" (Entrevista a joven rapero, Latin Fury, 2014), "generar una conciencia artística para que se inclinen más por la música y menos por la delincuencia" (Entrevista a joven rapero, Fundación Chocolate y Pan, 
2014), dan cuenta de las orientaciones implícitas en estas propuestas.

En estas narrativas es difícil precisar o identificar si aparece lo político, lo cultural o lo social como unidades fijas separadas. Como un riesgo a asumir, se plantea que en los anteriores enunciados convergen todas las dimensiones. Lo cultural encarna un barrio que ha sido objeto de valoraciones fuertes (estigmas), pero es el sujeto en el ejercicio de lo político quien busca borrar los imaginarios que afectan lo que realmente puede llegar a ser ese territorio. De la misma manera, el baile como forma de expresión humana, se relaciona con la intención de formar a alguien que más adelante pueda formar a otros; este es un ejemplo más de cómo lo político adquiere forma en otros espacios que son resignificados por los jóvenes.

Estos colectivos juveniles desarrollan también procesos de formación en temas centrales como: la objeción de conciencia, los derechos sexuales y reproductivos, el consumo responsable, el consumo de sustancias psicoactivas (SPA), los derechos humanos y la preservación del medio ambiente. Estos temas muestran cómo las ocasiones para actuar políticamente son cada vez mayores, en escenarios que someten a reflexión asuntos que antes eran más de carácter privado (como la sexualidad) y que buscan el pleno desarrollo de las capacidades humanas, la lucha contra cualquier forma de opresión y el compromiso con un sujeto de derechos.

\section{A MODO DE CIERRE}

Una categoría que permite precisar lo que está ocurriendo, es la subpolítica. Para Beck, Giddens y Lash (2002), el contexto de cambio que se ha señalado, donde el poder se desplazada del Estado hacia el mercado y la sociedad, genera las condiciones para la formación de actores que ahora participan al margen del sistema político y que buscan redefinir la política desde abajo.

La subpolítica, significa configurar la sociedad desde abajo. Visto desde arriba, esto tiene como consecuencia de la subpolitización, que grupos que hasta ahora no estaban implicados en el proceso de tecnificación e industrialización tengan cada vez más oportunidades de tener voz y participación en la organización de la sociedad (Beck, Giddens y Lash, 2002, p. 39).

Esto no significa de ninguna manera que estas prácticas políticas se den sin ningún tipo de restricciones económicas y políticas, porque de alguna manera, estas redes o mutualidades que los jóvenes construyen, surgen como luchas para hacerle contrapeso a múltiples formas de desigualdad. Por el contrario, significa que hoy es posible construir poder al margen de los escenarios convencionales donde se hace la política, porque se ha producido una descentralización del poder que ha trasladado la acción política a otros escenarios de la sociedad.

Esta idea planteada, se refleja en un último aspecto que parece ser una tendencia generalizada en todos los colectivos estudiados. La mayoría quiere expandir su trabajo a otros espacios o articularse con otras organizaciones. Frases como: "el objetivo de golpe natural es llegar a los barrios" (Entrevista a joven rapero, Golpe Natural, 2014) y "nosotros siempre buscamos expandirnos por todo lado" (Entrevista a joven rapera, Universal Rimas Colombia, 2014) muestran que hoy lo político adquiere forma en nuevos escenarios de socialización.

En el fondo, asistimos a la saturación de lo político, claro está que de otra forma; el de los microgrupos y microsolidaridades, o sea, nuevas formas de ver $y$ de participar en lo que se denomina la política, que precisamente vienen a llenar ese vacío que los partidos políticos y la política tradicional han dejado al no ser capaces de generar matrices discursivas que puedan interpelar a los jóvenes (Zarzuri, 2005, p. 7).

De esta manera, se está frente a una concepción de la socialización que se transforma en nuevas maneras de estar juntos. Hoy algunos procesos organizativos juveniles son menos rígidos y más flexibles. Ya no se trata de estructuras fijas vinculadas a objetivos homogéneos, 
con rangos de autoridad definidos, ni sistemas de acción coordinados de manera lineal. Se trata de un conjunto de redes de carácter informal, no estructurado y con escasa participación de grupos organizados. Estos colectivos construyen sentidos $y$ plantean demandas colectivas, que buscan una distribución equitativa de bienes materiales y simbólicos, a través de sistemas flexibles de acción que tienen la pretensión de darle otra direccionalidad a lo social.

\section{REFERENCIAS}

Beck, U., Giddens, A. y Lash, S. (2002). Modernización reflexiva. Politica, tradición y estética en el orden social moderno. Madrid, España: Alianza editorial.

Bourdieu, P. (2007). Estructuras, habitus, prácticas. El sentido práctico. Buenos Aires: Siglo xxi Editores.

Castaño, C. E. (2008). Para cartografiar la diversidad del@s jóvenes. Bogotá Dc: Instituto de Salud Pública.

Equipo ¿Bogotá cómo vamos? (2016). Informe de Calidad de Vida de Bogotá. Bogotá oc: Puntoaparte book versiting.

Escobar, A., Alvarez, S. y Dagnino, E. (2001). Política cultural \& cultura política. Una nueva mirada sobre los movimientos sociales latinoamericanos. Bogotá: Taurus-ICANH.

Garcés, A. (2007). Juventud música e identidad. Hip hop en Medellín. Educación cuerpo y ciudad: el cuerpo en las interacciones e instituciones sociales, 229-254

Giddens, A. (1997). Modernidad e identidad del yo. Barcelona: Península.

González, M., Aguilera, A. y Torres, A. (2014). Investigar subjetividades y formación de sujetos en $y$ con organizaciones $y$ movimientos sociales. En C. Piedraita, A. Díaz y P. Vommaro, Acercamientos metodológicos a la subjetivación política: debates latinoamericanos, 49-70. Bogotá: Cooperativa Editorial Magisterio.

Lechner, N. (1996). La Política ya no es lo que fue. Nueva Sociedad, 144, 104-113.

Lechner, N. (2002). Las sombras del mañana: la dimensión subjetiva de la política. Lom ediciones.

Navarrete, J. M. (2000). El muestreo en la investigación cualitativa. Investigaciones sociales, 4 (5), 165-180.

Perea, C. M. (2008) ¿Qué nos une?: jóvenes, cultura y ciudadanía. La Carreta.

Puyana, Y. (2013). Investigar en trabajo social desde relatos biográficos. En M. Himelda Ramírez, La investigación y la práctica en trabajo social, 111-137. Bogotá: Universidad Nacional de Colombia. Facultad de Ciencias Humanas.

Reguillo, R. (2000). Emergencia de culturas juveniles. Bogotá: Editorial Norma.

Restrepo, D. (2003). Las prácticas participativas: entre la socialización y la privatización de las políticas públicas. Enlaces y rupturas. Bogotá: Fundación para la Participación Comunitaria-Parcomún y Acción Ecuménica Sueca-Diakonía.

Touraine, A. (1993). Crítica de la modernidad. Madrid: Temas de hoy.

Zarzuri, R. (2005). Jóvenes, participación y movimientos sociales: hacia la construcción de nuevas formas de participación juvenil. Santiago: Centro de Estudios Socioculturales (cEsc).

Zemelman, H. (1989). De la historia a la política: la experiencia de América Latina. Siglo xxI.

Fecha de ingreso: 06/07/2017 Fecha de aprobación: 30/10/2017 
